\title{
Synthesis of aluminium nanoparticles by arc evaporation of an aluminium cathode surface
}

\author{
M GAZANFARI $^{1}$, M KARIMZADEH ${ }^{2, *}$, S GHORBANI $^{2}$, M R SADEGHI ${ }^{3}$, G AZIZI $^{2}$, \\ H KARIMI $^{2}$, N FATTAHI ${ }^{4}$ and Z KARIMZADEH ${ }^{4}$ \\ ${ }^{1}$ Department of Chemistry, Imam Hossein University, P.O. Box 16535-187, Tehran, Iran \\ ${ }^{2}$ Department of Chemistry, Isfahan University of Technology, P.O. Box 84156-83111, Isfahan, Iran \\ ${ }^{3}$ Department of Material Engineering, Isfahan University of Technology, P.O. Box 84156-83111, Isfahan, Iran \\ ${ }^{4}$ Department of Chemistry, College of Science, University of Mohaghegh, Ardabili, P.O. Box 56199-11367, \\ Ardabil, Iran
}

MS received 2 May 2013; revised 31 May 2013

\begin{abstract}
Aluminium nanoparticles (Al Nps) are synthesized using arc discharge method by applying direct current between aluminium electrodes in liquid environment without any use of vacuum equipment, heat exchangers, high temperatures furnaces and inert gases. After synthesis of $\mathrm{Al} \mathrm{Nps,} \mathrm{in} \mathrm{situ} \mathrm{coating} \mathrm{process} \mathrm{on}$ the nanoparticles was performed immediately. The effects of media on the yield and morphology of aluminium nanoparticles were investigated. Analysis result of the samples indicated that particle size was less than $30 \mathrm{~nm}$, when $120 \mathrm{~A} / \mathrm{cm}^{2}$ arc current was used. In addition, coating agent can affect arc velocity, arc stability, morphology and composition of the nanoparticles. Resultant nanoparticles were identified using $\mathrm{X}$-ray powder diffraction (XRD), also their surface morphology was studied by scanning electron microscopy (SEM) and transmission electron microscopy (TEM) and finally the accuracy of coating was assessed with infrared (IR) spectroscopy.
\end{abstract}

Keywords. Nano aluminium; arc discharge; scanning electron microscopy; transmission electron microscopy; X-ray diffractometer.

\section{Introduction}

Traditional energetic materials include single-molecular compounds such as TNT, RDX, HMX and TATB (Tyagi et al 2008). Although, these compounds are known to release their energy quickly, but the amount of energy that they release is restricted. By adding metal fuels such as magnesium, aluminium, boron, titanium and zirconium to energetic formulations, energy density can significantly be increased (Jouet et al 2005).

Synthesis and fabrication of new types of propellants containing nanometal powders are of great interest in the area of aerospace (Shevchenko et al 1994; Kuo 1997; Dokhan 2002; Kuznetsov 2003). Since conversion of aluminium to oxide form releases a lot of energy, this metal is considered to be special. Aluminium particles have been widely used in energetic materials and fuels because it is readily available and has the desired properties of a fuel in oxidation and reduction reactions such as high temperature combustion and flame. For example, addition of nanoparticles to a solid propellant improves density and specific impulse which are the most important factors

*Author for correspondence (morteza.karimzadeh@ch.iut.ac.ir) in many formulations. Micro-scale aluminium particles have been used previously. With advances in technology and materials processing, production of $\mathrm{Al}$ particles in the form of nano-scale particles improved both quantitatively and qualitatively and nowadays particles with a diameter of $100 \mathrm{~nm}$ and even less are produced. Reducing the particle size from micro- to nano-scale has effects on physical properties of $\mathrm{Al}$ particles. As particle size decreases, melting enthalpy and melting temperature are reduced. This is the effect of increasing surface area fraction of atoms with reduction in the particle size. Al Nps are valuable for many energy-related applications. Aluminium, because of its low price and high redox potential, is frequently used (Agrawal 1998; Haber and Buhro 1998; Kotov and Samatov 1999; Ermoline et al 2002; Kwon and Gromov 2002).

Nano aluminium particles are considered as hydrogen storage materials (Bald et al 2006). By increasing the specific surface area of nanoparticles, it is possible to complete the oxidation of nanoparticles. Although, reducing the size of nanoparticles is better, considering energy release, there are still problems with oxide layer. The layer of aluminium oxide is suitable for core stability of aluminium, but is considered as a dead weight and nearly represents $60 \%$ of the total mass of nanoparticles. 
The layer of aluminium oxide coatings are replaced with suitable stabilizers makes the aluminium more energetic. A number of publications which describe synthesis methods and protective coatings of aluminium have been investigated, but results show that aluminium content and stability of the particles decreased. Quan and Jacobsen (1995) synthesized Al Nps by evaporation method and used it for propulsion applications, but prepared nanoparticles were unspherical in shape. Ferrara et al (1988) used microwave plasma methods to synthesize Al Nps. Nanoparticles generated by this method were examined after they were aged for a long time. TEM images indicated that the average diameter of nanoprticles was $50 \mathrm{~nm}$, but particles as large as $500 \mathrm{~nm}$ were also present. Also Al Nps with broad size distribution (with diameters 10-500 nm) with various shapes were prepared by laser ablation method (Chen et al 2004). Application of this material as additives, propellants, fuels and explosives is under the influence of particle size and method of coating of Al Nps (Hunt et al 1998; Jiang and Yatsui 1998; Yavorovsky et al 2000; Romanova et al 2006). A variety of techniques, such as dynamic gas condensation, cryomelting process and plasma explosion process are also used to produce Al Nps (Vorozhtsov et al 2003; Sun and Simon 2007). In this paper, aluminium nanoparticles (Al Nps) are synthesized using arc discharge method by applying direct current between aluminium electrodes in liquid environment without any use of vacuum equipment, heat exchangers, high temperature furnaces and inert gases.

\section{Experimental}

Arc method was used to produce nanoparticles described in this work. Power supply with specific voltage and current was purchased from Bam Electronic and aluminium rods were purchased from Paya. Polyethylene glycol 300, ethylene glycol, distilled water and salt were all supplied by Sigma-Aldrich. The apparatus used for synthesizing coated-Al Nps is schematically shown in figure 1.

This system required only a direct current (d.c.) power supply and commercially available aluminium rods. Hence, two high-pure aluminium rods (92.5\%) with diameter of $2.5 \mathrm{~mm}$ and length of $40 \mathrm{~mm}$ were used as a movable anode and a static cathode in our arc discharge experiments. Electrodes were placed in various media with specific angles. Separating the anode from the cathode increases the voltage, and a high electric current (120 A/ $\mathrm{cm}^{2}$ ) passes through between two electrodes. It tends to vaporize aluminium atoms from the anode electrode surface. Cooling the aluminium vapour results in the production of primary particles which turns into Al Nps dispersed in the media by nucleation mechanism. Then, major part of the product was observed as sediment in the container floor and a small amount was observed as supernatant liquid. This experiment was performed in polyethylene glycol, ethylene glycol, distilled water with a concentration of 0.15 of $\mathrm{NaCl}$ and tap water with a concentration of 0.25 of $\mathrm{NaCl}$. Resultant product was collected with the use of filtration and centrifugation, and was placed in $350{ }^{\circ} \mathrm{C}$ furnace temperature for $2 \mathrm{~h}$. After drying, the resultant particles were analysed. The experimental conditions employed have relative advantages of being economical, simple and use of available starting materials. Nanoparticles characterizations were carried out using X-ray diffraction (XRD), scanning electron microscopy (SEM), transmission electron microscopy (TEM). Infrared (IR) spectroscopy analysis showed important adsorption of formed bonds.

\section{Results and discussion}

The goal is to find the best media for fabrication of $\mathrm{Al}$ Nps with better homogeneity, good particle size distribution and higher yield.

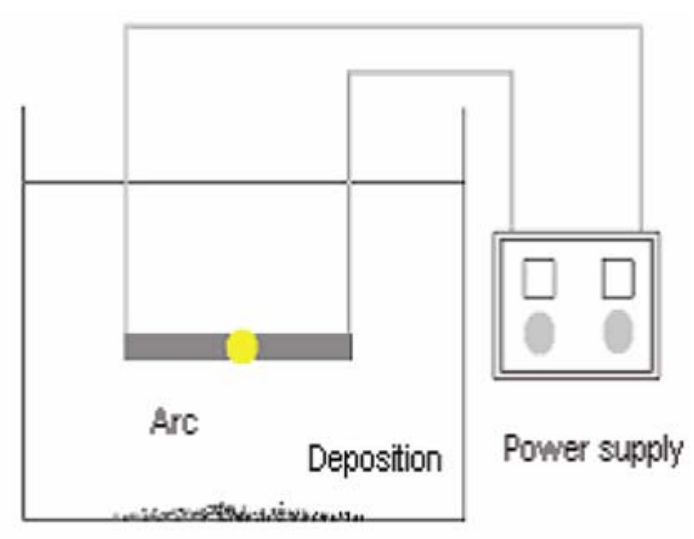

Figure 1. Schematic of experimental setup.

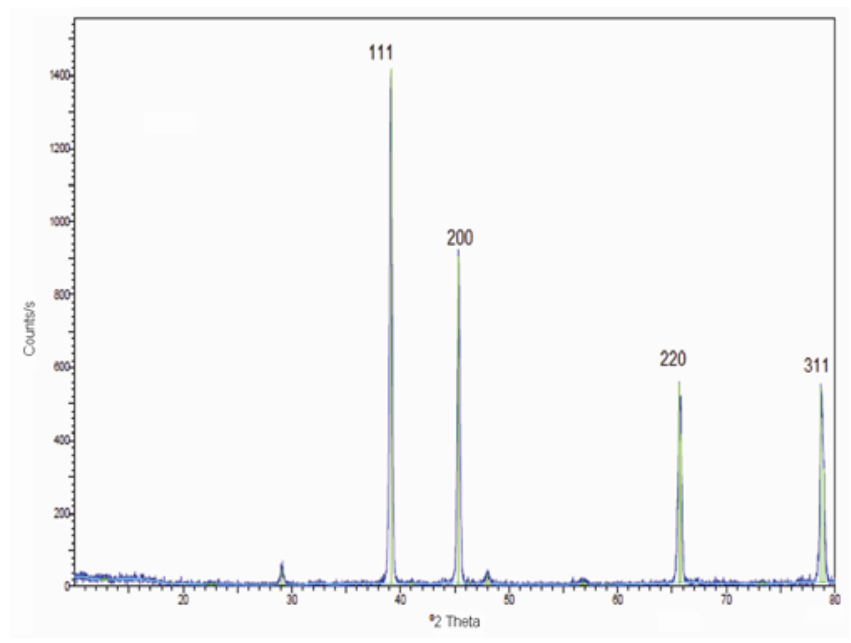

Figure 2. XRD pattern of nanoparticles obtained after $\mathrm{Al}$ electrodes are subjected to arc discharge. 

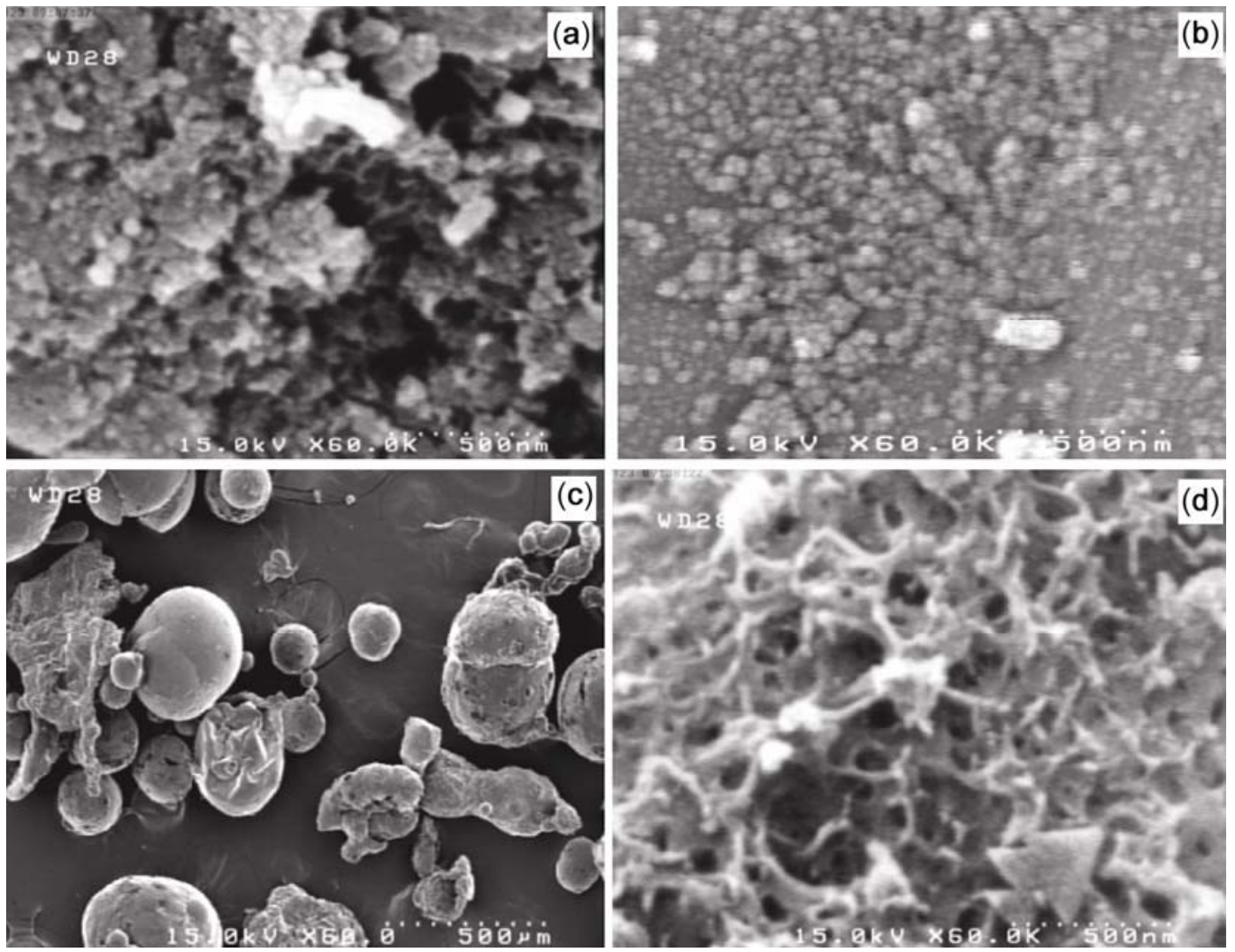

Figure 3. SEM images of Al Nps in (a) distilled water; (b) ethylene glycol; (c) polyethylene glycol 300 and (d) tap water.

The shape of our synthesized nanoparticles was spherical with an average size in the range of $10-90 \mathrm{~nm}$ measured by SEM and TEM. As shown, these particles are not agglomerated specially in ethylene glycol (EG) medium. It is comparable or in some cases having superior yield than in other methods. Already it is noted that drawback to produce $\mathrm{Al} \mathrm{Nps}$ is that aluminium exposed to air forms a passivating oxide layer. Not only does the oxide inhibit combustion, but also it prevents the strategy of reducing particle size, by consuming an increasing portion of the particle mass, and thus energy density. The method used here gives clearance about production of aluminium nanoparticles in organic and liquid media that are completely free of oxide and XRD shows it very well. In order to examine the oxidative stability of the coated particles, we exposed the coated aluminium to heat in oven. When the oven temperature is increased to $350{ }^{\circ} \mathrm{C}$, no change has been detected. This suggests that the coating successfully prevented $\mathrm{Al} \mathrm{Nps}$ from oxidizing up to at least $350{ }^{\circ} \mathrm{C}$. Because of this, we do all the characterizations after drying the nanoparticles in $350{ }^{\circ} \mathrm{C}$. It is necessary to say that these particles show good stability after aging for a long time (one year). These media coat efficiently unoxidized aluminium surface and provides an effective barrier to air oxidation at temperature up to $350{ }^{\circ} \mathrm{C}$.

\subsection{XRD analysis of products}

To determine the chemical composition and structure of produced particles, X-ray diffraction (XRD) technique was used for the obtained particles in the ethylene glycol media. The main peaks of XRD pattern were compared with the peaks related to Al Nps in the databases. Perfect match between the pattern of the sample with a reference pattern illustrates the formation of $\mathrm{Al} \mathrm{Nps}$ and the same combination of components with nanoaluminium products. The only difference between the reference pattern with the pattern of the sample was the broad peaks in the spectrum of the samples which is further evidence for the nano-scale particles. Based on Williamson-Hall equation, width of XRD peaks is due to two factors: size and stress. If the particles are smaller, peaks will be wider. The corresponding XRD pattern of deposits obtained from experiments with ethylene glycol is shown in figure 2. Nanoparticles are characterized using a Holland Philips Xpert X-ray powder diffraction (XRD) diffractometer (CuK $\alpha=0 \cdot 9$, radiation, $\lambda=0 \cdot 154056 \mathrm{~nm}$ ), at a scanning speed of $2^{\circ} / \mathrm{min}$ from 0 to $80^{\circ}(2 \theta)$. As seen, this pattern has four sharp peaks. Four characteristic peaks for aluminium $\left(2 \theta=39 \cdot 1,45 \cdot 1,65 \cdot 6\right.$ and $\left.78 \cdot 2^{\circ}\right)$, corresponding to Miller indices (111), (200), (220) and (311), were observed. This revealed that the resultant particles were 

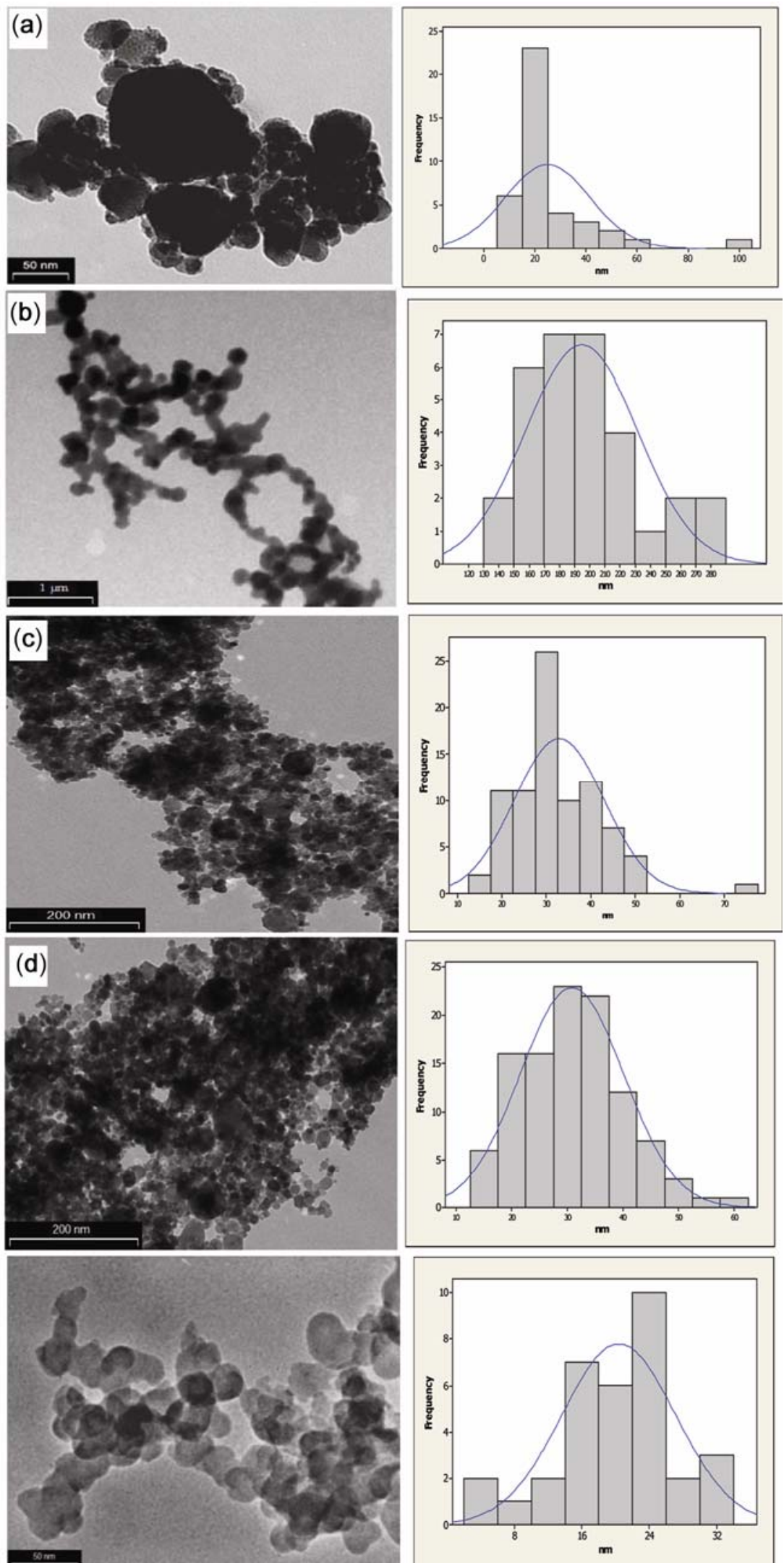

Figure 4. TEM micrographs and diameter distribution histograms for Al NPs for four samples with average diameters as follows: TEM images of Al Nps in (a) tap water, $25 \mathrm{~nm}$, (b) polyethylene glycol, $195 \mathrm{~nm}$, (c) distilled water, $32 \mathrm{~nm}$ and (d) ethylene glycol, $20 \mathrm{~nm}$. 
pure face centred cubic ( $f c c$ ) aluminium. Also, using Scherrer equation and data of XRD pattern, nano-sized particles of $30 \mathrm{~nm}$ was obtained. In the desired pattern, there is no peak related to the aluminium oxide. Therefore, EG coats Al Nps and protects their surface from oxidation to aluminium oxide.

\subsection{SEM analysis of products}

Obtained particles were analysed using scanning electron microscopy in different media in order to ensure that they are in nano-scale size and to determine their surface

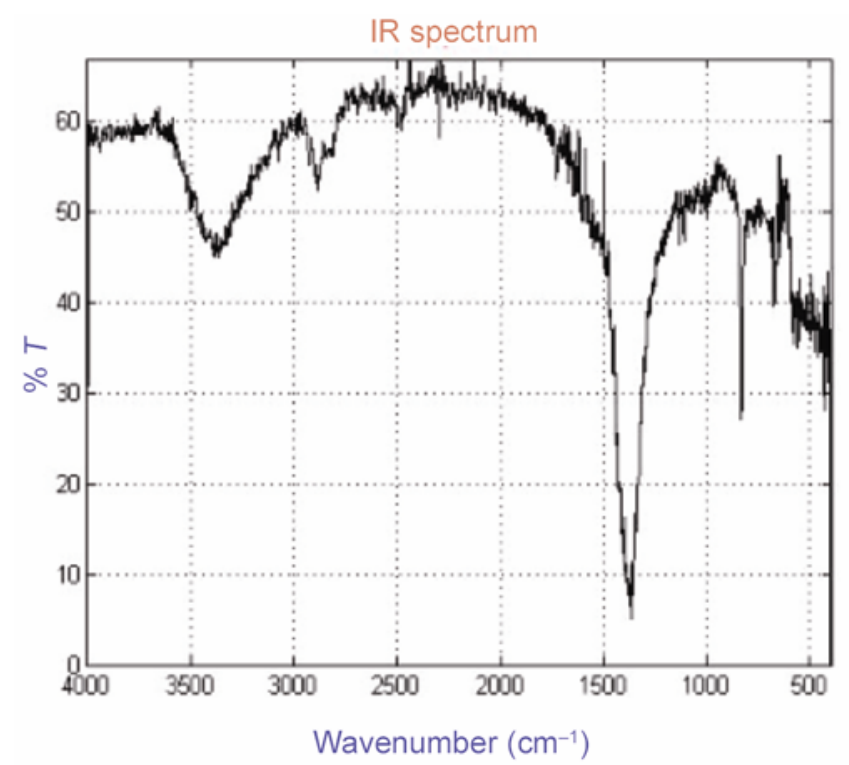

Figure 5. IR spectrum of Al Nps coated with ethylene glycol.

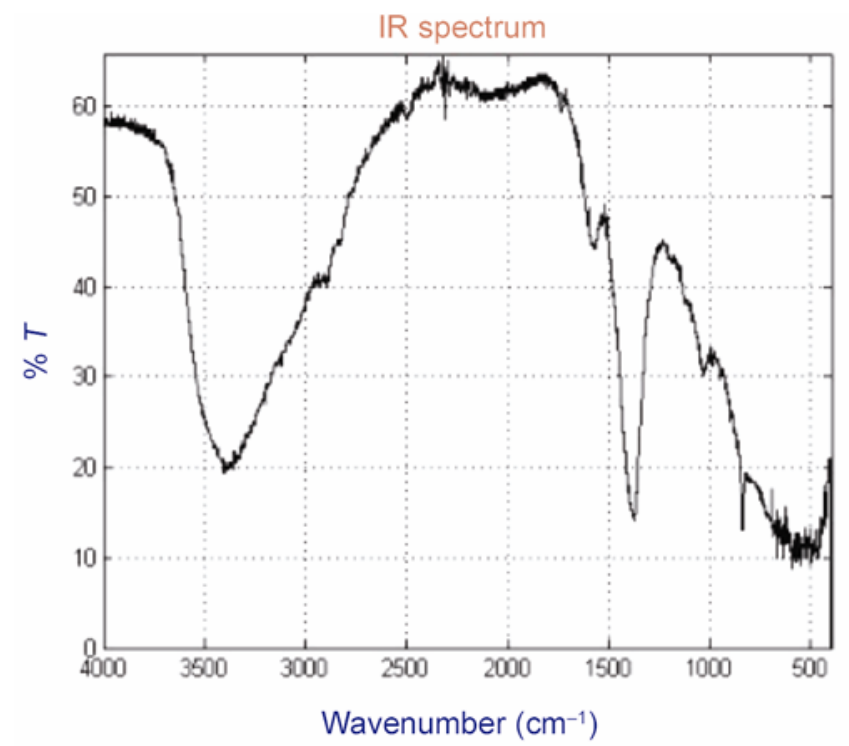

Figure 6. IR spectrum of Al Nps coated with distilled water. morphology. Particles were in nano-scale dimensions and were well separated from each other in ethylene glycol media. Particles morphology was spherical. Particle size was obtained between 8 and $40 \mathrm{~nm}$ in this media. Particle morphology was similar to bee's nest in distilled water and they were with little concentration, also particle size was obtained between 15 and $60 \mathrm{~nm}$. But, particle morphology was obtained as a sponge in tap water and particle size was between 10 and $60 \mathrm{~nm}$. It should be noted that nanoparticles were not synthesized in polyethylene glycol 300. The accumulation of particles was very high and particles with micro size were fabricated (figures 3a-d).

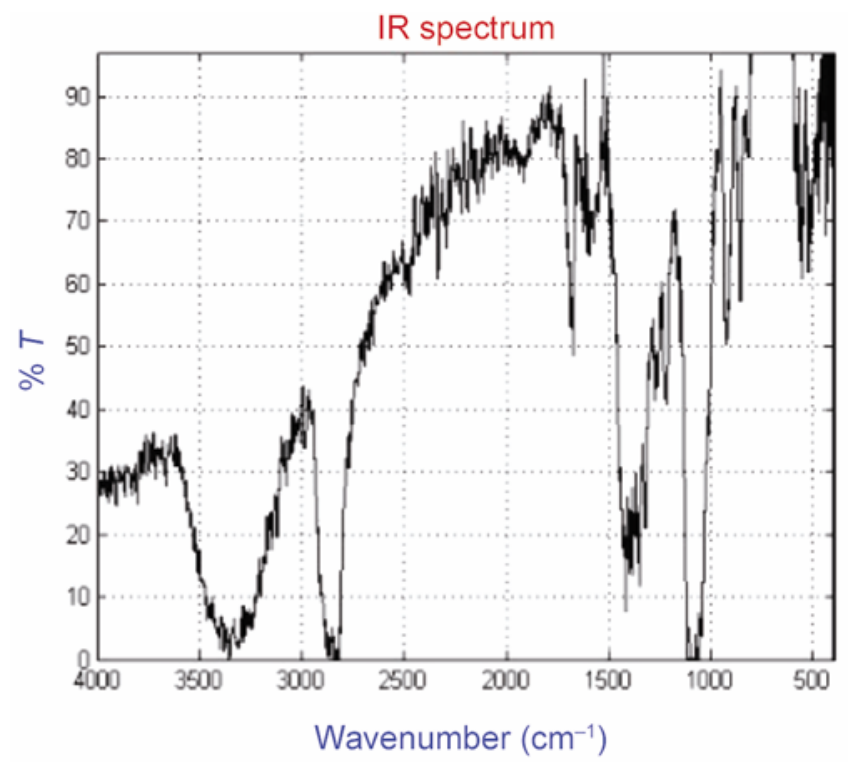

Figure 7. IR spectrum of Al Nps coated with polyethylene glycol 300.

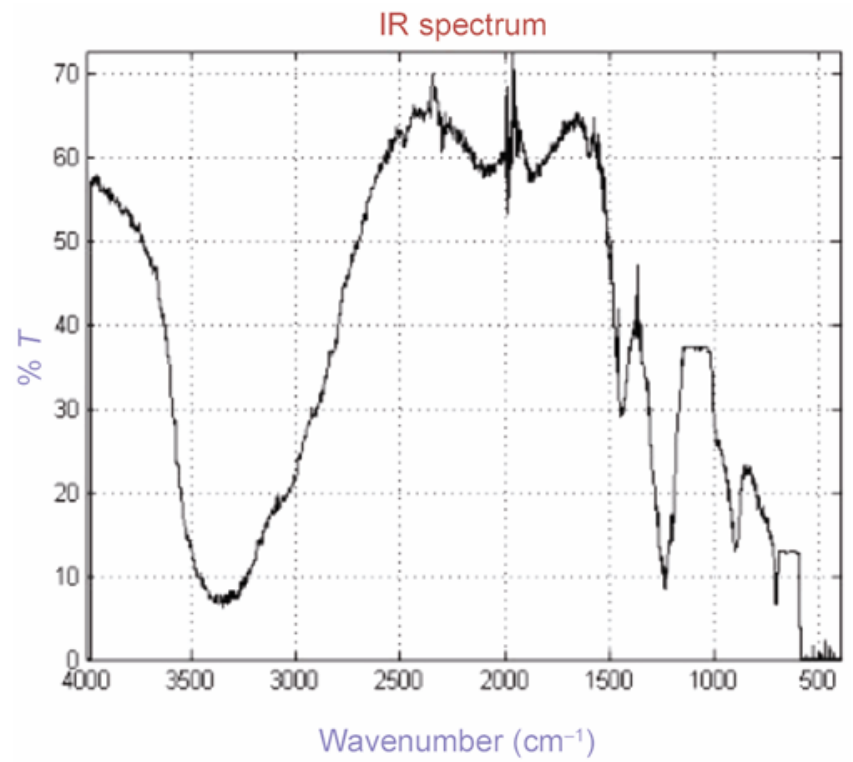

Figure 8. IR spectrum of Al Nps coated with tap water. 


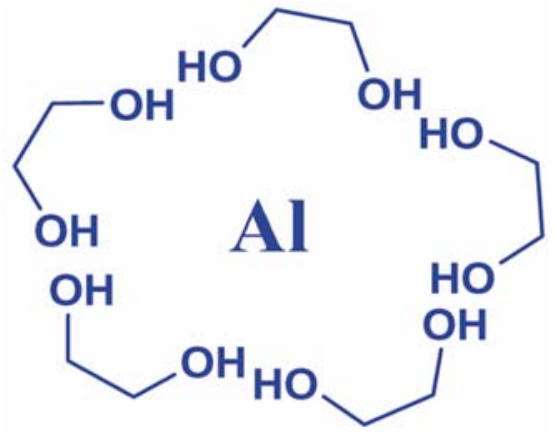

Figure 9. Illustration of Al Nps coated with ethylene glycol.

\subsection{TEM analysis of products}

Analysis of TEM images along with their histograms provided information with respect to the size distribution of particles produced in each medium. TEM photograph of particles is presented in figure 4. Sponge-like particles produced in tap water that was shown in SEM image (figure 3d) was in good agreement with obtained TEM image (figure 4a). TEM image shows particles are in different sizes. In this image, not only large number of nanoparticles with a size of $20-30 \mathrm{~nm}$ is seen, but also few particles with larger sizes (around $60 \mathrm{~nm}$ and $100 \mathrm{~nm}$ ) have been seen. It should be noted that when this medium is used, good dispersion has not been achieved and agglomeration of nanoparticles was found in the system. The mean particle size of the tap water coated Al Nps ranged from 20 to $30 \mathrm{~nm}$ and its histogram represented it to very well. Figure 4(b) shows morphology of the particles in polyethylene glycol (PEG) and its histogram indicates that the size of the particles is about $0 \cdot 2 \mu \mathrm{m}$. TEM micrograph of the particles in distilled water (figure 4c) indicates a spherical particle shape like a wasp nest with sizes in the range of $15-60 \mathrm{~nm}$. Also, it must be noted that the particles in EG media were much smaller $(8-40 \mathrm{~nm})$ and more uniform than those obtained in distilled water and specially PEG media, and histogram based on TEM analysis (figure 4d) shows it very well.

\subsection{IR analysis of products}

Al Nps are rapidly oxidized due to their high chemical activity. To overcome this problem, various reagents can be used to trap particles and thus, prevent the oxidation of nanoparticles. These reagents not only prevent the oxidation of Al Nps, but also prevent from sticking the particles together (their growth as a mass of particles) and thus, reduce the particle size. In this work, four different reagents were used which act as a liquid media and particle stabilizer. In each experiment, infrared (IR) spectroscopy was performed for the obtained samples to verify coating of nanoparticles. IR spectra corresponding to each of the solvents is given in figures 5-8.
Broad peak in each spectrum corresponds to $\mathrm{OH}$ functional group, which reacts with the obtained Al Nps. This functional group created good coating on nanoparticles to prevent oxidation as shown in figure 9.

\section{Conclusions}

A process for the synthesis of coated-aluminium nanoparticles was presented. Al Nps was synthesized by arc evaporation of an aluminium cathode surface. The stability of Al Nps was discussed in order to prevent oxidation. XRD, SEM, TEM and IR analyses revealed that the ethylene glycol coating is chemically bonded to the $\mathrm{Al}$ Nps. Ethylene glycol was selected as the best media for the synthesis of nanoparticles among other liquid media. Al Nps produced by this way is very cheap and affordable compared to other methods.

\section{References}

Agrawal J P 1998 Prog. Energ. Combust. 241

Bald C P, Hereijgers B P C and Bitter J H 2006 Angew. Chem. Int. Ed. 453501

Chen T Y, Omnes L, Vaisserman J and Doppelt P 2004 Inorg. Chim. Acta 3571299

Dokhan A 2002 The effects of aluminum particle sizes on aluminized propellant combustion. Ph.D. dissertation, Georgia Institute of Technology, Georgia

Ermoline A, Schoenitz M and Dreizin E 2002 Nanotechnology 13638

Ferrara J D, Tessier-Youngs C and Youngs W J 1988 Inorg. Chem. 272201

Haber J A and Buhro W 1998 J. Am. Chem. Soc. 12010847

Hunt E M, Hampikian J M, Poker D B and Evans N D 1998 (Switzerland: Elsevier) vol. 31, p. 409

Jiang W and Yatsui K 1998 Plasma Sci. 261488

Jouet R J, Warren A and Rosenberg D 2005 Chem. Mater. 17 2987

Kotov Y A and Samatov O M 1999 Production of nanometer-sized ain powders by the exploding wire method (USA: Elsevier) vol. 12, p. 119

Kuo K K 1997 Challenges in propellants and combustion 100 years after Nobel (New York: Begell House 636)

Kwon Y and Gromov A A 2002 Combust. Flame 131349

Kuznetsov V T 2003 Propellants Explos. Pyrotech. 283019

Quan L Z and Jacobsen R W 1995 J. Am. Chem. Soc. 117 5889

Romanova V M, Pikuz S A, Mingaleev A R and Shelkovenko T A 2006 J. Phys. Soc. 56 B349

Shevchenko V G, Kononenko V I, Latosh I N, Chupova I A and Lukin N V 1994 Combust. Explo. Shock. 30635

Sun J and Simon S L 2007 Thermochim. Acta 46332

Tyagi H, Phelan P and Prasher R 2008 Nano Lett. 81410

Vorozhtsov A B, Ivanov Y F, Osmonoliev M N, Sedoi V S, Arkhipov V A, Bondarchuk S S, Korotkikh A G and Kuznetsov V T 2003 Propellants Explos. Pyrotech. 283019

Yavorovsky N A, Domashenko V G and Balukhtin P V 2000 Sci. Technol. USA 3280 